Zusammenfassend lä $\beta t$ sich sagen, daß die Dehydrodoisynolsäure in Einzeldosen von 2,5 mg und Gesamtdosen bis zu $15 \mathrm{mg}$ sehr gut vertragen wurde. Es gelang bei Kastratinnen mit $10 \mathrm{mg}$ histologisch verifizierte Proliferationsphasen zu erzeugen. Des weiteren kann das neue Östrogen höchstwahrscheinlich auf alle für die Follikelhormonbehandlung in Frage kommenden Indikationen erstreckt werden. Besonders hervorgehoben werden muB seine perorale Wirksamkeit.

R. WENNER Und C. A. JÖ̈L.

Universitäts-Frauenklinik(Dir.: Prof, Dr.TH. KoLLER). Basel, den 13. März 1945.

\section{Räumliche Reizsummation der helladaptierten Netzhaut}

Die folgenden Gesetzmäßigkeiten haben sich während Studien über das Gesichtsfeld ergeben. Bei der Gesichtsfeldaufnahme wird $z u$ einem gegebenen Schwellenreiz jener Ort im Gesichtsfeld gesucht, an dem der Reiz gerade wahrgenommen wird. Der Schwellenreiz wird definiert durch Feldgröße $(F)$ des Reizobjektes und durch den Quotienten aus Objekthelligkeit minus Grundhelligkeit: Grundhelligkeit $(\Delta I / I)$, den Kontrast. Das Objekt wurde mit ca. $5^{0}$ pro Sekunde Geschwindigkeit aus der Gesichtsfeldperipherie hereingefüht. Bei Helladaptation (1,6-50 asb. Grundhelligkeit untersucht) gilt für farblose Objekte 2 wischen 6,5 und 52 ' über weite Gebiete der Netzhaut mit großer Annäherung folgende Gesetzmäßigkeit :

1. Jedes Flächenelement des Reizobjektes trägt zum Schwellenreiz proportional seiner Leuchtdichte bei.

2. Bei verschieden großen Reizobjekten gilt weitgehend $\Phi=\left(\frac{F_{0}}{F}\right)^{0,86}$, worin $\Phi$ eine $\mathrm{Zahl}$ ist, welche angibt, auf welchen Teil der Kontrast vermindert werden mul (z. B. bei projizierten Marken durch Graufilter), um trotz Objektvergrößerung von $F_{\mathrm{a}}$ auf $F$ das gleiche Gesichtsfeld zu erhalten. Es besteht also eine unvollkommene Summation.

Die beiden Gesetzmäßigkeiten werden durch die Tabelle illustriert. AI und BI zeigen zwei Reizobjekte, in denen, von ihrem Zentrum ausgehend, die Leuchtdichte sich verschieden ändert. Dementsprechend muB bei Objektvergrößerung in verschiedener Weise die Gesamtleuchtdichte herabgesetzt werden (AII und BII). Rechnet man nach Satz 1 beide Objekte auf gleichmäBige Leuchtdichte um, so erhält man Werte (CII, a und $b$ ), die gut untereinander und mit den aus Gleichung $\Phi=\left({\frac{F}{F^{0}}}^{0,86}\right)$ errechneten Werten (CIII) übereinstimmen.

Die beschriebene Gesetzmäßigkeit gilt außerhalb der Netzhautmitte. Die Gültigkeitsgrenze gegen die Netzhautmitte hin liegt um so peripherer, je größer das Reizobjekt ist. Sie wird für jede Objektgröße, wie es scheint, durch den Ort bestimmt, wo zwei winzige Objekte, welche einen gegenseitigen Abstand gleich dem Durchmesser des betrachteten Reizobjektes haben, bei zunehmender Leuchtdichte sofori als zwei Objekte erkannt werden, wenn man sie aus der Peripherie gegen die Gesichtsfeldmitte bewegt. "Summation" und "Distinktion" stehen in peripheren Netzhautpartien demnach in einem ge wissen "Entweder-oder"-Verhältnis. Für das helladap tierte Auge gilt also in dem angegebenen Gebiet weder das Riccosche Gesetz ( $I \cdot F=$ konst.) noch das PIPERsche Gesetz $(I \cdot \sqrt{I}=$ konst.) noch auch das PIERONsche Gesetz für die Fovea $(I \cdot F 0,3==$ konst. $)$.

Ausführliche Mitteilung demnächst in der Zeitschrift "Ophthalmologica".

H. Goldmann

Universitäts-Augenklinik, Bern, den 13. März 1945.

Käumliche Reizsummation dey helladaptiertun Notzhat

\begin{tabular}{|c|c|c|c|c|c|c|}
\hline \multicolumn{3}{|r|}{ Reizobjektgröle (Durchmesser) } & $6, b^{\prime}$ & $13^{\prime}$ & $26^{\prime}$ & $5 y^{\prime}$ \\
\hline \multirow[t]{2}{*}{ A } & \multicolumn{2}{|c|}{$\begin{array}{l}\text { Lichtstärke des Reizobjektes (ohne } \\
\text { Filter) in relat. Maß } . . .\end{array}$} & 1 & 4 & 13,4 & 58,5 \\
\hline & \multicolumn{2}{|c|}{ II $\Phi$ (Mittel von 11 Personen) . . } & 1 & $0,31 \pm 0,0044$ & $0,1 \pm 0,002$ & $0,033 \pm 0,003$ \\
\hline \multirow[t]{2}{*}{$\mathrm{B}$} & \multicolumn{2}{|c|}{ I wie AI . . . . . . . . . . } & 1 & 4,7 & 20,2 & 101 \\
\hline & \multicolumn{2}{|c|}{ II $\Phi$ (Mittel von 4 Personen) . . } & 1 & 0,242 & 0,077 & 0,022 \\
\hline \multirow[t]{4}{*}{$\mathrm{C}$} & \multicolumn{2}{|c|}{$\begin{array}{l}\text { I Lichtstärke bei homogener Leucht- } \\
\text { dichte des Objektes }\end{array}$} & 1 & 1 & 16 & 64 \\
\hline & & a) $\Phi$ aus AII berechnet . . . & 1 & 0,31 & 0,088 & 0,033 \\
\hline & & b) $\Phi$ aus BII berechnet . . . & 1 & 0,31 & 0,086 & 0,026 \\
\hline & \multicolumn{2}{|c|}{ III aus $\Phi=\left(\frac{F_{0}}{F}\right)^{0,86}$ berechnet . . } & 1 & 0,304 & 0,092 & 0,028 \\
\hline
\end{tabular}

